Bol. Acad. peru. leng. 55. 2013 (37-56)

\title{
EL ESPAÑOL DE COSTA RICA
}

\section{L'ESPAGNOL DU COSTA RICA}

\section{THE SPANISH OF COSTA RICA}

\author{
Estrella Cartín de Guier \\ Academia Costarricense de la Lengua
}

\section{Resumen:}

Primeros estudios sobre el tema: don Carlos Gagini (Diccionario de costarriqueñismos). Aportes importantes: Arturo Agüero, Víctor Arroyo, Víctor Sánchez, Miguel Ángel Quesada. Rasgos del español de Costa Rica, que sin ser privativos de nuestra variedad lingüística, son de uso frecuente y configuran la identidad del español de Costa Rica. Señalamiento de estos rasgos en los campos de la fonética, de la morfosintaxis y del léxico. En el campo fonético, entre otros: seseo, yeísmo, deshiatización, pronunciación ápico alveolar de la "t" en el grupo "tr". En el morfológico: diminutivos (ico-ica), reduplicación intensiva del diminutivo y del superlativo. El voseo en el léxico, léxico costarricense no usado en España. Léxico hispánico con significado particular en Costa Rica.

\section{Résumé:}

Premières recherches sur le sujet: don Carlos Gagini (Diccionario de costarriqueñismos). Contributions importantes: Arturo Agüero, Víctor Arroyo, Víctor Sánchez, Miguel Ángel Quesada. Traits de l'espagnol du 
Costa Rica, qui sans être exclusifs à notre variété de langue, sont d'un emploi fréquent et constituent l'identité de l'espagnol du Costa Rica. Relevé de ces traits dans les domaines phonétique, morphosyntaxique et lexical. Dans le domaine phonétique, entre autres: prononciation de "c" et "z" comme "s" (seseo), prononciation de ll comme y (yeísmo), diérèse, prononciation apico-alvéolaire du " $t$ " dans le groupe " $t r$ ". Dans le domaine morphologique: diminutifs (ico-ica), redoublement intensif du diminutif et du superlatif. Dans le lexique, emploi du "vos", lexique du Costa Rica non employé en Espagne. Lexique hispanique avec une signification particulière au Costa Rica.

\section{Abstract:}

Early studies on the subject. Don Carlos Gagini (Dictionary of Costa Rican terms). Major contributions: Arturo Agüero, Víctor Arroyo, Víctor Sánchez, Miguel Ángel Quesada. Features of the Spanish in Costa Rica, which without being exclusive in our linguistic variety, are nevertheless of frequent use and shape the identity of the Spanish in Costa Rica. Markings of these features in the fields of phonetics, morphosyntax and vocabulary. In the field of phonetics for example we have the "seseo" (special employment of the "s"), the semiconsonant " $y$ " (yeismo), the loss of hiatus, the apicoalveolar pronunciation of the " $t$ " in the cluster "tr". At the morphological level we have the diminutive endings "icoica", intense reduplication of the diminutive and the superlative. The use of "vos" in the vocabulary, Costa Rican lexical terms not used in Spain. Spanish terms with special meaning in Costa Rica.

Palabras clave: lingüística; español; Costa Rica; oralidad; escritura.

Mots clés: linguistique; espagnol; Costa Rica; oralité; écriture.

Key words: linguistics; Spanish; Costa Rica; orality; spelling.

Fecha de recepción:

Fecha de aceptación:
$28 / 02 / 2013$

$22 / 04 / 2013$ 
El primer autor que exploró los campos del español costarricense fue don Carlos Gagini. Su primera obra de mérito fue el Diccionario de barbarismos y provincianismos de Costa Rica, publicado en 1893.

Aunque esta obra (según lo reconoce el propio autor) no era muy completa, fue de gran importancia y sirvió de punto de partida para obras posteriores. Este diccionario recogió las voces de origen indígena americano usadas en nuestro país y no registradas en diccionarios castellanos, voces no insertas en los léxicos de nuestra lengua y palabras castizas usadas con otras acepciones, errores prosódicos y ortográficos más comunes, arcaísmos con actualidad en la lengua popular costarricense, extranjerismos, modismos y refranes.

Ya don Carlos contó con algunas obras de consulta como las Apuntaciones críticas de Cuervo, el Diccionario de chilenismos de Rodríguez, el de Voces cubanas de Pichardo y el de Peruanismos de Rivodó, al igual que con los estudios de Batres Jáuregui.

En 1918, Gagini publicó la segunda edición de su diccionario, con enmiendas y considerablemente aumentado. Esta vez llevó el título de Diccionario de costarriqueñismos.

A los trabajos de Gagini siguen importantes investigaciones sobre el tema como las de don Arturo Agüero, don Víctor Arroyo, don Víctor Sánchez, don Miguel Ángel Quesada y otros.

Los rasgos de nuestra variedad lingüística que señalaré a continuación tienen validez particularmente en el habla del Valle Central de Costa Rica y regiones aledañas.

Haré referencia a algunos fenómenos de nuestra lengua en los campos de la fonética, la morfología y el léxico. Veamos en primer término algunos rasgos fonéticos del español de Costa Rica. En relación con las vocales puede anotarse: 
1. Confusión de timbre entre vocales inacentuadas. Como en la mayoría de los casos se trata de formas antiguas, estas no son exclusivas de Costa Rica. Veamos algunos casos:

a) Cambio de "e" por "i"

En el habla rural y popular es frecuente oír "centura", "enteligente" y "polecía”. Pero aun entre la gente culta se oye "bacenilla" por "bacinilla", "comelón” por "comilón” y "molenillo" por "molinillo".

En una obra de nuestro autor costumbrista Aquileo Echeverría, en una de sus "Concherías", "Modelo epistolar", leemos:

Ayer me trujo Jacinto

La ropilla,

Los cigarros

Y su carta y la cajita

Con ungüento de soldao.

Ayer mesmo me lo unté;

De viaje se atarantaron;

Esta mañana me vide

Y ni uno vivo ha quedao.

b) Cambio de "i” por "e"

Ocurre con cierta frecuencia como es el caso de "disvariar" en lugar de "desvariar" y de "dispertar" en vez de "despertar". En la obra del mismo autor anteriormente citado leemos:

Salúdeme a ña Prudencia,

Lo mesmo que a los muchachos,

$\mathrm{Y}$ no me olvide, que yo

Me paso en usted pensando.

Soy su novio y servidor

Pedro Vindas

Posdata

Perdone los dos borrones

Pero jue que me meniaron. 
c) Cambio de "o" por "u"

Es frecuente el cambio. Aun entre personas cultas oímos decir "ruciar" por "rociar", "revulucionario" por "revolucionario", "almuhada" por "almohada". En la obra de Aquileo encontramos:

Como mi almuada es de paja

Y mi novia no está vieja

Toda la noche la paso

Con la paja tras la oreja.

Muchos de los casos obedecen a la fonética combinatoria: asimilación, disimilación, aféresis, epéntesis; y otros, a la analogía o a la confusión de prefijos que es también analógica como el caso de "desgañotarse" en lugar de "desgañitarse" por analogía con "gañote", "destornillarse de risa" en lugar de "desternillarse de risa" por analogía con "tornillo".

2. Son frecuentes los casos de aféresis, tanto de vocal como de sílaba. Es el caso de "cequia" por "acequia", "ora" por "ahora", "maca" por "hamaca".

En "La vela de un angelito" dice Aquileo:

—iViva Domingo!

—iVivan ñor José y Gabriela!

— iVivan los dueños de la casa!

—iOtro trago pa l'orquesta!

En otro pasaje leemos:

Solo mama no está bien

Porque la sigue fregando

El dolor en el cuadril

La tos, el pujo y el flato;

Por suerte está mejorcita

Con solo la miel de palo,

Con güitite y alcanfor

Bol. Acad. peru. leng. 55(55), 2013 
Que le aplicó mano Pablo

De ayer pa'cá se levanta

Unque no sale del cuarto.

Y en otro pasaje dice:

Onde Jacinto hubo baile

Pal estreno de un retablo

Muy lujoso que trajeron

El domingo de Cartago

Pa meter a Santa Rita

y al Señor Resucitao.

3. Es frecuente el uso de vocal epentética. El campesino dice "haberá" por "habrá"; además, es de uso casi general "tembeleque" en lugar de "tembleque", y "culeca" en vez de "clueca".

4. El diptongo "ie" en posición inicial de palabra, cuya $i$ debe pronunciarse como "yod" semiconsonante suele exagerarse hasta $y$, aun en la pronunciación de personas educadas. Decimos "yelo", "yel", "yerba"; y no "hielo", "hiel", "hierba". Esta pronunciación se extiende a voces derivadas de las anteriores, aunque el diptongo no tenga acento; por ejemplo, decimos "desyerbar", "yerbicida".

5. El grupo "ea" se transforma en "ia". Decimos: "pasiar", "tiatro". En el caso de los infinitivos, la sinéresis la cometen las personas cultas cuando hablan familiarmente.

6. El hiato "eo" se convierte generalmente en diptongo. En el habla familiar decimos "pion" por "peón", "lion” por "león”.

7. El grupo "oa” pasa al diptongo "wa”. Por ejemplo "cuágulo" por "coágulo", "Juaquín” por Joaquín. Las personas cultas, por lo general, pronuncian el grupo pero no en dos sílabas sino en una; dicen "Joa-quín" y no "Jo-a-quín". 
8. En el grupo "oe" sucede lo mismo que en el anterior y se oye decir "pueta”, "puesía” y "cuhete” en lugar de "poeta”, "poesía” y "cohete".

Señalaré, a continuación, algunos fenómenos relacionados con las consonantes.

En Costa Rica está totalmente generalizado el "seseo" y el "yeísmo". Pronunciamos la z como s y la ll como $y$. De igual manera se dice "calló" (de callar) que "cayó" (de caer); "halla" (de hallar) que "haya" (de haber).

9. La "t" es ápico alveolar en el grupo "tr", especialmente en la región central del país. Decimos "tres", "cuatro", "tremendo".

10. La "d" ápicodentointerdental fricativa sonora normalmente se pronuncia de esta manera, pero en ciertas posiciones tiende a desaparecer como en el caso de los morfemas de participios que terminan en ado e ido y al final de palabra ("casao", "pesao", "usté”, "verdá").

Veamos un ejemplo de "Las Concherías":

En la misa del domingo

Hubo dos amonestaos

Ramón Cerdas con Gregoria

Y Cirila con ñor Campo.

Dicen que Ramón se casa

Pal primer jueves de mayo

Me contó José María

Que ayer lo vido encalando

Y qu'él mismo le contó

Que ya'bía comprao los trastos

Y qui'hace dos meses tiene

Dos chanchillos amarraos

Diez chompipes, dos gallinas

$\mathrm{Y}$ un motico y diez carracos

Dichosotes los que tienen 
Tata rico y patrón macho.

¿Sabe que se le murió

El gallo cuijen el sábado?

Le empezó como un ronqueo

Cantaba desentonao

Se le cayeron las plumas

Se le pandió el espinazo;

Ayer lo encontraron tieso

iPobrecillo, tan buen gallo!

En España es normal hasta en el habla culta pronunciar "mercao", "soldao"; pero en Costa Rica y el resto de Hispanoamérica, cuando la conversación no es informal, se dice "mercado", "soldado".

11. La "g" posdorsovelar oclusiva sonora suele desaparecer en el grupo "gn" y es frecuente oír pronunciaciones como "Inacio", "inorante", sobre todo en la lengua rural; sin embargo, aun las personas cultas suelen decir "persinarse" por "persignarse".

12. La " $\mathrm{r}$ " ápico alveolar vibrante sonora múltiple que se escribe $r$ o $r$, en la región central del país no es vibrante sino fricativa ("rico", "perro", "enredadera”). Esta es una característica de pronunciación muy notable que distingue a los costarricenses del Valle Central de los de la provincia de Guanacaste, donde sí se pronuncia la $r$ nítidamente vibrante.

13. La " $r$ " ápico alveolar sonora simple delante de pausa es pronunciada por los costarricenses del interior del país con un sonido como fricativo ensordecido o totalmente sordo ("cantar", "coser", “amor”). Es esta una pronunciación muy característica.

14. El grupo "tr". En el Valle Central y en los lugares a donde se ha extendido su población, la $t$ alveolar, asimilada por la $r$ siguiente que no es vibrante sino fricativa, origina con esta una especie de sonido africativo, alveolar y sonoro o ensordecido a veces ("traer", "cuatro", "trapear"). Es este un rasgo dialectal muy acentuado aunque no sea exclusivo de Costa Rica. 
15. El grupo “dr". Es frecuente, aunque no general, la supresión de la $d$ que va delante de la $r$ y precedida de $n$, o sea, $n d r$. Ejemplo: "Anrés", "Alejanro". Es esta una pronunciación muy común en el habla informal.

Se dan también matátesis, casi todas coincidentes con otros países de habla española. Es el caso de "itsmo" (istmo), "pachotada" (patochada), "charramasca" (charamasca), "cabrestro" (cabestro), entre otras.

\section{MORFOLOGÍA}

En este campo me limitaré a señalar algunos aspectos que pueden caracterizar mejor nuestra variedad lingüística como es el empleo de diminutivos y superlativos; el voseo; y el uso de algunas preposiciones, adverbios e interjecciones.

Diminutivos. El uso de diminutivos es muy frecuente en Costa Rica, sobre todo en el lenguaje familiar de todas las esferas sociales. Se usa de preferencia el sufijo ito-ita. Raras veces se oye cito-cita. Se dice "casita", "caminito", "piedrita". Con este sufijo se forman diminutivos de sustantivos, adjetivos, adverbios, participios y gerundios. Se dice "Fernandito", "cosita", "vidita”, "blanquito", "pequeñito", "inutilito", "nadita”, "naditica”, "suyita”. Es frecuente la expresión "por vida suyita”.

Ispiamos para un guarumo

Pa unos itabos, inadita!

Pa la poza, el agua clara

Como si juera llovida;

La perra seguía ladrando

$\mathrm{Y}$ en la mesma desusidia.

Dijo Canuto: quizás

Se le habrá clavao espina;

Le reparamos las patas,

La panza, la rabadilla

El pescuezo, las orejas

Hasta el rabo, inaditica! 
Y en otro pasaje:

Mama le manda memorias

Tata, Luis y los muchachos.

No deje de persinase

Pa que no lo tiente el malo,

Porque dicen qu'en Heredia

Es onde hay sesenta rayos

Por vida suyititica.

Es frecuente el diminutivo de adverbios, participios y gerundios tales como "ahorita”, "cerquita”, "acostadito”, “escondidito”, “callandito”, "corriendito".

A los costarricenses nos apodan "ticos" y aun nosotros mismos nos llamamos así porque en la conversación familiar decimos, por ejemplo, "zapatico" en lugar de "zapatito", "poquitico" en vez de "poquitito". Como puede notarse, no es tico-tica lo que se agrega sino ico-ica por ito-ita.

Intensifica este fenómeno y lo hace más frecuente la duplicación intensiva o expresiva del diminutivo en ito ("chiquito", "chiquitico", "chiquititico"). En la conversación familiar, esta duplicación intensiva se vuelve reduplicación. Ejemplo: "poquitico", "poquititico" y hasta "poquitititico". Se dice, también, "pobrecitititico".

En la formación del superlativo, el sufijo ísimo-ísima tiene mucha vitalidad y es muy usado. Es frecuente en Costa Rica la reduplicación intensiva del superlativo casi tanto como la del diminutivo. Decimos "pobrísimo", "pobrisísimo" y "pobresisisisimo"; "guapísimo”, "guapisísimo" y "guapisisisisimo".

El voseo. Una de las características del español nuestro es el uso del voseo, el que sin ser un hecho exclusivo es empleado por personas de todas las clases sociales, sin excepción, como pronombre de segunda persona singular. El uso de vos en vez de tú y de ti junto con la mezcla de te en lugar de os con el posesivo tu constituye un fenómeno idiomático desarrollado exclusivamente en América. Es el que señala Cuervo cuando dice que 
una de las manifestaciones en el castellano de aquí es la que arrancando del antiguo uso español de los pronombres de segunda persona, ha conservado el uso de vos con las inflexiones "tomas", "comes" "hicistes", ocasionando el olvido de tú, ti, vosotros y dando origen a una nueva inflexión ("vos tomabas", "tenías") con la mezcolanza de vos y te ("te engañas", "ya veréis que nada he dicho de vos"); hecho muy extenso que da al habla familiar y popular un aspecto completamente distinto del castellano.

El vos es un arcaísmo hispánico que se consolidó y evolucionó al margen del español peninsular. En el Nuevo Mundo, el paradigma del tuteo se fundió con el del vos medieval de donde surgió el voseo americano. El empleo de vos en lugar de tú es rasgo peculiar en ciertas regiones de Hispanoamérica.

En Costa Rica el voseo es general. Se usa en todos los estratos sociales y abarca todo el territorio nacional. El tuteo es ajeno al costarricense. El uso de tú es uno de los indicios de extranjería. El tratamiento de tú por parte de un costarricense en la conversación familiar sería visto como afectación y cursilería. No se da en nuestro país la coexistencia de tú y vos. Se usa predominantemente el vos. Es el tratamiento entre amigos y parientes aunque no siempre con reciprocidad, pues suele ocurrir que un pariente o amigo de mayor edad vosee al más joven y este trate a aquellos de usted. Generalmente, los padres vosean a los hijos y lo normal es que los hijos traten de usted a los padres, sobre todo en las zonas rurales donde todavía se considera irrespetuoso vosear a los mayores.

Los esposos se vosean recíprocamente, aunque hay excepciones y algunos se tratan de usted, sobre todo en los medios rurales en los que se conserva este tratamiento de respeto.

Los novios de las ciudades se vosean casi en su totalidad. Los jóvenes de ambos sexos, aun cuando no exista mucha amistad ni confianza entre ellos, acostumbran vosearse, sobre todo en los medios urbanos. Lo mismo sucede entre estudiantes de ambos sexos. Cada día se generaliza más el voseo y las nuevas generaciones vosean a los mayores sin tener en cuenta edad o rango, sin guardar consideración ni respeto alguno. 
Cabe señalar que el posesivo de vos no es vuestro-vuestra sino tuyo-tuya-tuyos-tuyas. En Costa Rica, al igual que en las regiones donde se vosea, no se usa vosotros para dirigirse a las personas que son tratadas de vos porque el plural de vos es ustedes.

En el uso de los adverbios es frecuente el costarriqueñismo "acuanta" ("ha poco tiempo"). Decimos "secedio acuanta", "acuantacito"; o sea, que acaba de suceder. Este uso ha quedado relegado a las comunidades rurales.

Es frecuente oír la expresión "de un pronto a otro" para indicar algo que sucede de pronto.

Se utiliza en Costa Rica la expresión "al propio" en lugar de "adrede" o "a propósito". Por ejemplo: "Usted quebró ese florero al propio".

La preposición "hasta" por lo general se usa de manera inapropiada. Se dice: "El parque abre hasta las diez" para indicar que "El parque no abre sino hasta las diez". Por otro lado, se emplea "entre" en lugar de "cuanto". Ejemplo: "Entre más grita, menos logra su meta".

Son empleadas en nuestro país ciertas interjecciones que le resultarían extrañas a un español y a la mayoría de los hablantes hispanoamericanos, tales como: "adio", "idiay" y "achara".

"Adio" expresa extrañeza y equivale a "qué va”, "qué es eso”. En "Visita de pésame" de Aquileo Echevarría leemos:

- ¿Y qué jue eso de Gaspar?

Pa Reyes lo vi en la iglesia

Y estaba gordo, alentao.

Antantier llega manuela:

¿No sabes quién se murió?

iÑor Gaspar!

—iAdió! ¿De veras?

- Si murió como a las doce.

Mañana a las diez lo entierran. 
En otro pasaje encontramos:

-Que le vaya bien.

-Mil gracias.

- Trele el caballo, Drilo.

—iAdió! Si me vine a pata.

"Idiay" es una interjección que se origina en "y de ahí" y equivale a "y bien”, "pues”, “¿y qué?”.

Veámosla en la literatura, en "Comprando ayotes":

Hast ayer, como le digo

Que bin'un bandido chancho

Di un vecino y al vijiar

Qu'en Misa Mayor andábamos

$\mathrm{Y}$ que el perro que tenemos

Estaba bien amarrao

Se dio gusto con los seles

Y si a tiempo no llegamos

Se atoya los desasones

- ¿Y diay nada reclamaron?

-Nada. P'alivio de males

El chanchillo es del cuñao

Un hombre que es malo, bueno

Y el mismo patas con guaro.

"Achara" es una interjección que equivale a "iqué lastima!". Es frecuente usar "iacharita!". Veamos un ejemplo de "Visita de pésame":

-L'espero p'al novenario

- Yo no puedo por mi pierna;

Pero vendrán las muchachas.

-Achara que usté no pueda

Porque va a estar muy alegre.

Tata mercó una ternera

$\mathrm{Y}$ tres garrafas de guaro 
$Y$ seis frascos de mistela,

Y además a contratao

Cuatro músicos de Heredia;

Y pa'los misterios tiene

Cohetes de luz y bombetas.

Ya usté le conoce el genio.

¡Cuando se raja es de veras!

\section{LÉXICO}

Al igual que en los demás países hispanoamericanos, el léxico del español de Costa Rica está compuesto por más de un $90 \%$ de palabras hispánicas, de manera tal que no se puede afirmar que tengamos un léxico autóctono elevadísimo que llegue a causar problemas de comunicación entre los países.

No obstante, recuerdo siempre una de mis primeras salidas por las calles de Madrid al iniciar mis estudios universitarios en esa ciudad. Luego de ver en una tienda algunas prendas que me interesaron ingresé al negocio y pregunté: “¿Cuánto valen esas enaguas, esa cartera y esa faja que están en la ventana?”. El empleado me miró sin entender y preguntó a su compañero, quien respondió: "Me parece que la chica desea saber el precio de la falda, el bolso y el cinturón que están en la vitrina”. O sea que yo no había acertado ni con una sola palabra.

Además del léxico panhispánico, podemos distinguir también el léxico usado en Costa Rica y en otros países hispanoamericanos, pero no en España. Por ejemplo: "cartera”, "celular”, "cacho” (por "cuerno”), "estampilla", "saco" (por "chaqueta”).

Existe también el léxico hispánico con significado particular en nuestro país o en muy pocos países del área.

Ejemplos:

Botellita. Cada uno de los mesocarpios de los cítricos donde se almacena el jugo. 
Desmorecerse. Faltar la respiración a causa de la risa o del llanto. Despachador. Se dice de una persona poco hospitalaria o de un asiento incómodo.

A la par. En vez de "al lado".

Pena. Con el significado de "vergüenza".

Campo. En lugar de "espacio" o "sitio".

La expresión "ni hablar" no posee la connotación negativa que se le da en España o en países sudamericanos. Significa lo contrario, es algo estupendo.

Tenemos también el léxico de origen indígena. Debido al carácter tardío de la conquista de Costa Rica, los primeros pobladores de este país ya manejaban las palabras que el español americano había incorporado; estos términos pertenecían a las lenguas antillanas ("cacique", "huracán", "papaya”, "guayaba") y aztecas ("tomate”, "guacal”, "jícara”). Hay otras palabras como "súrtuba" y "purruja” que probablemente provienen del huetar.

Por otra parte, la influencia de hablas hispanas extranacionales dejó su huella en el habla costarricense; en particular la lengua mexicana que, sin lugar a dudas, ha sido la de mayor influencia.

De entre las posibles explicaciones para el fenómeno de la influencia mexicana en Centroamérica, estarían en primer lugar la evidente vecindad geográfica, o una serie de afinidades que van de lo étnico a lo histórico y que tendrían que ver no solo con las migraciones precolombinas, sino también con la conquista, la época colonial y la fugaz incorporación al imperio de Iturbide, cuando recién se proclamó la independencia.

En lo concerniente a Costa Rica, tal influencia emerge con mayor evidencia a mediados del siglo pasado. La sociedad costarricense urbana o rural se deleita con las películas de los cómicos mexicanos y en la vida campesina calan muy hondo la canción ranchera y la figura del charro. Esta impronta cultural supone también cambios lingüísticos que se van a manifestar en el componente léxico. 
Existen en el léxico de nuestra variedad lingüística mexicanismos muy arraigados tales como "maje", "mariachi", "pachuco" y "pura vida". Haré referencia a dos que forman parte del habla cotidiana del costarricense, especialmente de los jóvenes: "maje" y "pura vida".

A diario oímos un saludo como el siguiente: "Idiay, maje, quiubo ¿pura vida?". Y la respuesta: "Pura vida, maje. ¿Y vos?".

La palabra "maje" procede del español de México y se encuentra registrada en la mayoría de los países centroamericanos con el valor de "tonto". En Costa Rica se ha convertido, principalmente, en un pasillo de la Escuela de Estudios Generales de la Universidad de Costa Rica. "Dos estudiantes conversaban y uno le dijo al otro: 'Mae, ¿vistes qué mae más mae ese mae'. Y el otro le respondió: 'Sí, mae, bien mae”. Nótese la pluralidad semántica y las diversas funciones que desempeña el vocablo. Es, en primer término, un vocativo, una forma de apelación: "mae". Luego es usado como sustantivo: "viste qué mae" ("viste qué tío o qué tipo"), y pasa a ser adjetivo: "más mae” ("más tonto"), y de nuevo sustantivo: "ese mae" ("ese tipo").

El vocablo en sus inicios forma parte del lenguaje del hampa y la marginalidad y luego por una transferencia sociolectal recibe la marca de jerga juvenil. Con la palabra "maje" se forman frases coloquiales muy usadas, tales como "agarrar de maje" ("coger de tonto") y "hacerse el maje" ("hacerse el tonto"). Si bien es cierto que este uso revela cierta creatividad y originalidad, limita, sin lugar a dudas, las posibilidades expresivas del joven; además, es lenguaje que contribuye al deterioro de la palabra.

La otra expresión que merece ser comentada es: "pura vida". La fuente irradiadora de este préstamo fue la película mexicana titulada Pura vida. El protagonista Melquiades utiliza esta unidad pluriverbal trece veces en el transcurso del film.

En el español de Costa Rica esta unidad fraseológica es de uso relativamente reciente, pues se registra aproximadamente en la década de los 
ochenta. Es usada como forma de saludo. Se oye a diario, especialmente entre jóvenes:

—Idiay, qué ipura vida?

-Pura vida.

Se emplea como locución adjetiva para indicar que algo es simpático, agradable, bueno, bonito. Ejemplo: "Esa mujer es pura vida".

— ¿Qué te pareció el concierto?

—iPura vida!

—¿iTe gustaría salir mañana de paseo?

—Claro que sí. iPura vida!

Se usa para indicar estado anímico:

— ¿Cómo estás? ¿Bien?

-Bien.

- ¿Pura vida?

-Pura vida.

Para referirse al estado de salud:

—¿Estuviste enfermo?

—Sí, pero ya estoy pura vida.

Es evidente la categoría de neologismo de la unidad fraseológica "pura vida" en el español de Costa Rica, pues solamente se encuentra documentada en obras lexicográficas de reciente publicación.

Para finalizar, quisiera referirme a un término muy nuestro y muy usado en Costa Rica: el vocablo "chunche".

El "chunche", como dice don Alberto Cañas, en Costa Rica lo es todo. Es la palabra que nos sirve a los costarricenses para designar 
todas las cosas, tanto aquellas cuyo nombre ignoramos como las que no recordamos. Las piezas de la mecánica, por ejemplo, raras veces las nombramos; un piñón, un pivote, una soldadura son "chunches". El que va a comprar un tirabuzón o un sacacorchos, probablemente pedirá "un chunche de abrir latas".

Se ha tratado de interpretar este uso y se ha querido encontrar en esta práctica un rasgo de nuestra idiosincrasia. Don Alberto Cañas lo considera un síntoma de timidez. El costarricense antes de decir el nombre exacto de algún objeto nuevo, por timidez ante la posibilidad de que los otros lo juzguen como usuario de un lenguaje demasiado culto, opta por designar el objeto con el nombre de "chunche". Evitará así despertar desconfianza, ya que alguien demasiado culto en el hablar, de maneras demasiado refinadas, termina por ser mal visto e inspira desconfianza. Y es que quien no diga "chunche" será porque se siente superior y eso no se perdona en Costa Rica; ya que los costarricenses nos sentimos todos iguales y vivimos en función de ello. Lo que no se permite es el airecillo de superioridad. La palabra "chunche" es nuestra y señal de igualitarismo. A pesar de que no hay acuerdo académico que le dé la bienvenida al "chunche", este se va colando. Con cada nueva palabra que el diccionario acepta, acepta al mismo tiempo la palabra "chunche" que es sinónimo de todas.

Espero haber logrado esbozar un perfil de esta variedad lingüística que nos identifica y configura frente al mundo, al mismo tiempo que da cuenta de nuestra peripecia vital y es fiel reflejo del modo existencial de nuestra comunidad. Ese tesoro que heredamos de nuestros conquistadores quienes, como dice Neruda, "se llevaron el oro y nos dejaron el oro...”. Se lo llevaron todo y nos dejaron todo. Nos dejaron las palabras. 


\section{BIBLIOGRAFÍA}

AGÜERO CAVES, Arturo. El español de Costa Rica. San José de Costa Rica, Editorial Universidad de Costa Rica, 2009.

ALONSO, Amado. Estudios lingüísticos. Temas hispanoamericanos. Biblioteca Románica Hispánica. Madrid, Editorial Gredos, 1953.

ALONSO, Dámaso. "Unidad y defensa del idioma". En: Memoria del II Congreso de Academias de la Lengua Española. Madrid, 1956.

ARROYO, Víctor Manuel. El babla popular en la literatura costarricense. Publicaciones de la Universidad de Costa Rica, 1971.

BONILLA, Abelardo. Historia y antología de la literatura costarricense. Imprenta de Trejos Hermanos, 1957.

ECHEVERRÍA, Aquileo J. Concherías. San José de Costa Rica, Editorial Universitaria, Imprenta Trejos Hermanos, 1953.

QUESADA PACHECO, Miguel Ángel. Actitudes hacia el habla campesina de Costa Rica a través de la historia. Herencia 2. Universidad de Costa Rica, 1990.

. Una nueva perspectiva en torno al habla popular. Herencia 10. Universidad de Costa Rica, 1990.

. Nuevo diccionario de costarriqueñismos. Cartago, Editorial Tecnológica de Costa Rica, 1991.

. "Pequeño atlas lingüístico de Costa Rica". En: Revista de Filología y Lingüística. 18 (2), Universidad de Costa Rica, 1942.

. "El español de América Central". En: M. ALVAR (dir.). Manual de dialectología hispánico. El español de América. Barcelona, Ariel, 1996.

Bol. Acad. peru. leng. 55(55), 2013 
ROSEMBLAT, Ángel. La lengua y la cultura de Hispanoamérica. Tendencias actuales. Paris-Toulusse, Librairie des Editions Espagnoles, 1951.

SÁNCHEZ CORRALES, Víctor Manuel. "Comunicación de masas y emigración léxica. Del cine clásico mexicano a la conformación de la costarriqueñidad". En: Kañina. Revista de Artes y Letras, Universidad de Costa Rica, XXXIV, 2010.

. "Fricación de erre en el español de Costa Rica: un caso de escisión fonológica”. En: Revista de Filología y Lingüística. 12 (2), Universidad de Costa Rica, 1985.

Maje. De la denotación a la apelación. Instituto de Investigaciones Lingüísticas, Universidad de Costa Rica.

\section{Correspondencia:}

\section{Estrella Cartín de Guier}

Presidenta de la Academia Costarricense de la Lengua.

Correo electrónico: academia@ad.ac.cr 